\title{
SUPERNOVA 1987 A
}

\section{André Maeder, Geneva}

\author{
(Geneva Observatory)
}

Since the Big-Bang, supernovae (SN) have been the most violent events observed in the Universe - stars explode and may become as bright as $10^{10}$ Suns. On 24 February 1987, a new supernova designated SN 1987 A was discovered with the naked eye by I. Shelton, from the Las Campanas Observatory in Chile, in the Large Magellanic Cloud, a satellite galaxy of ours located at some 50000 parsec $\left(1.5 \times 10^{18} \mathrm{~km}\right)$ from the Sun. Immediately it was recognized as an event of tremendous astrophysical importance, being the first SN visible without instruments since the year 1604 and offering an unprecedented opportunity for us to study stellar evolution, nucleosynthesis and the birth of a neutron star.

As soon as they learned of the event, astronomers and particle physicists rushed to their detectors. Fortunately the neutrino burst from SN 1987 A had already been recorded in underground experiments, and round the clock ground-based observations began in the radio and optical wavelengths. From space, observations in the UV were performed by the IUE satellite, the IR by the Kuiper airborne IR Observatory, X-rays by the Ginga satellite, $\mathrm{X}$ - and $\gamma$-rays by the Russian space station Mir. The $\gamma$-rays were also observed by the SMM satellite and by balloon transported detectors. The interest in the scientific community was so large that on $26 \mathrm{Fe}$ bruary a second explosion started: that of astronomical papers devoted to $\mathrm{SN}$ 1987 A!

\section{Historical Supernovae and Extragalac- tic Surveys}

The study of historical chronicles has shown that eight events among the temporary "guest stars" recorded over the last 2000 years can reliably be considered as supernovae. In all cases, the radio observations of the supernova remnant has confirmed the identification. The $\mathrm{SN}$ of 1054 is the most famous as it gave rise to the Crab Nebula with its central pulsar, while the last record stems from 1604 when Kepler made a careful description of the event.
Our best information on $\mathrm{SN}$ statistics comes from extragalactic surveys which show that for a spiral galaxy like ours, an average of $2.2 \mathrm{SN} /$ century are to be expected. The discrepancy between the eight cases actually recorded and the $40-50$ to be normally expected is mainly due to the very strong interstellar absorption within the galactic plane, where most $\mathrm{SN}$ are likely to occur.

The surveys also show that supernovae are essentially of two types, called SNI and SNII. SNI attain up to $10^{10}$ times the solar luminosity $\left(L_{\odot}\right)$, show no hydrogen, and occur in any kind of galaxy, to mention just a few of their properties. They are convincingly modelled [1, 2] by accreting white dwarfs in binary systems, which, owing to mass transfer, undergo gravitational collapse. SNII show more scatter in their behaviour and reach up to $3 \times 10^{8} L_{\odot}$; their spectra exhibit hydrogen lines and they occur only in spiral galaxies. They are interpreted as resulting from the explosion of a massive star in the red supergiant stage.

\section{The Surprising Blue Precursor}

SN 1987 A has been firmly classified as an SNII because of the hydrogen lines observed, but at its appearance it had many puzzling properties. After a month of controversial debate, the precursor could be firmly identified as the star Sk -69 202 in the Large Magellanic Cloud. Strikingly, instead of being a red supergiant with a radius of, say, $800 R_{\odot}$, Sk -69202 had been a blue supergiant with a radius of about $40 R_{\odot}$ and an initial mass estimated to be about $20 M_{\odot}$. The optical and spectroscopic properties of SN 1987 A were also quite unusual: first of all, its brightness was "only" $5 \times 10^{7}$ $L_{\odot}$, while the ejection velocities, estimated from the blue shifted component of absorption lines, were quite fast: up to $30000 \mathrm{~km} / \mathrm{s}$ instead of $5000-10000$ $\mathrm{km} / \mathrm{s}$ for a standard SNII. SN $1987 \mathrm{~A}$ also experienced within the first week a very steep decline (by a factor $10^{3}$ ) in its UV luminosity.

These various pecularities found their explanation in terms of a smaller initial radius of the progenitor star. A smaller star has denser outer layers slowing down the leakage of photons through the expanding envelope and diminishing the luminosity. At emergence, the shock wave had travelled a shorter distance and had retained a higher velocity and as the initial explosion went faster, cooling also was faster with the consequent steep initial decline in UV intensity.

But why had the precursor a smaller radius? Together with the much discussed neutrinos seen in the Mont Blanc experiment, this was and is still the most disputed aspect of SN $1987 \mathrm{~A}$. The star probably went through the normal evolutionary stages of a $20 \mathrm{M}_{\odot}$ star with a hydrogen burning phase lasting 8.8 million years. Then during the central helium burning phase (1.2 million years) it became a red supergiant, following which it could become a star of smaller radius in one of two ways. First, it could lose most of its envelope through the stellar winds, shown recently to act intensively on red supergiants, and become a blue supergiant with a smaller radius. The difficulty with this interpretation is that the light curve of $\mathrm{SN}$ 1987 A during the first 120 days can only be modelled if the star has retained some 5 to $10 M_{\odot}$ of its initial hydrogen rich envelope $[3,4]$. The other solution is that in addition to a moderate mass loss, the precursor star experienced an efficient mixing process bringing much helium into the outer layers $[3,4]$. With a less opaque envelope, the star gets hotter and becomes a blue supergiant. Although this possibility is very attractive, the hydrodynamical process responsible for such a mixing has not yet been identified. Spectroscopic observations of blue supergiants in the Large Magellanic Cloud, i.e. sisters of Sk -69 202, may provide useful information in the future.

\section{Evolution Towards Central Collapse}

Paradoxically, the sequence of events occurring at the stellar centre during evolution is less uncertain than what happened at the surface. Models show that the inner layers of a star are conditioned by a succession of nuclear reac- 
Table 1 - Main nuclear burning phase in the course of the evolution of a $20 M_{\odot}$ star

\begin{tabular}{|l|c|c|}
\hline \multicolumn{1}{|c|}{ Reaction } & $\begin{array}{c}\text { Tempera- } \\
\text { ture, K }\end{array}$ & Time \\
\hline $\mathrm{H} \rightarrow{ }^{4} \mathrm{He}$ & $(2-6) \times 10^{7}$ & $8.8 \times 10^{6} \mathrm{a}$ \\
${ }^{4} \mathrm{He} \rightarrow{ }^{12} \mathrm{C},{ }^{16} \mathrm{O}$ & $(1-4) \times 10^{8}$ & $1.3 \times 10^{6} \mathrm{a}$ \\
${ }^{12} \mathrm{C} \rightarrow{ }^{16} \mathrm{O},{ }^{20} \mathrm{Ne},{ }^{24} \mathrm{Mg}$ & $(5-8) \times 10^{8}$ & $8 \times 10^{3} \mathrm{a}$ \\
${ }^{16} \mathrm{O} \rightarrow{ }^{28} \mathrm{Si},{ }^{31} \mathrm{P},{ }^{32} \mathrm{~S}$ & $1.5 \times 10^{9}$ & months \\
${ }^{28} \mathrm{Si} \rightarrow{ }^{56} \mathrm{Fe}$ & $(2-4) \times 10^{9}$ & days \\
\hline
\end{tabular}

tions (cf Table 1) which occur at higher and higher temperature $T$. These reactions lead to the so-called onion skin model: close to the centre, elements are synthetized at higher $T$ and in regions of much higher densities, up to $10^{10} \mathrm{~kg} /$ $\mathrm{dm}^{3}$.

Like a good runner, the star starts slowly and, as seen in Table 1, strongly accelerates at the end, the lifetimes of the various nuclear stages becoming shorter and shorter. One reason is that less and less nuclear energy is available per mass unit at each step. More importantly, neutrino emission mainly by $\mathrm{e}^{+} \mathrm{e}^{-}$pair annihilation, the cross-section for which rises with energy, becomes enormous and instantaneously removes all the energy produced at the stellar centre, so speeding up the evolution. The neutrino luminosity $L_{v}$ may become up to $10^{5}$ times the photonic luminosity.

Losing its energy and having little nuclear fuel, the iron core starts collapsing. When $T \cong 10^{10} \mathrm{~K}$ and $\rho \cong 10^{10} \mathrm{~kg} / \mathrm{dm}^{3}$, two other effects precipitate an accelerating central implosion. First, the Fermi energy of the electrons becomes larger than the mass difference between neutrons and protons and the neutronisation reaction $\mathrm{p}+\mathrm{e}^{-} \rightarrow \mathrm{n}+\mathrm{v}$ occurs, producing neutrons and also removing the electrons, the pressure of which was supporting the core. Second, the pho-

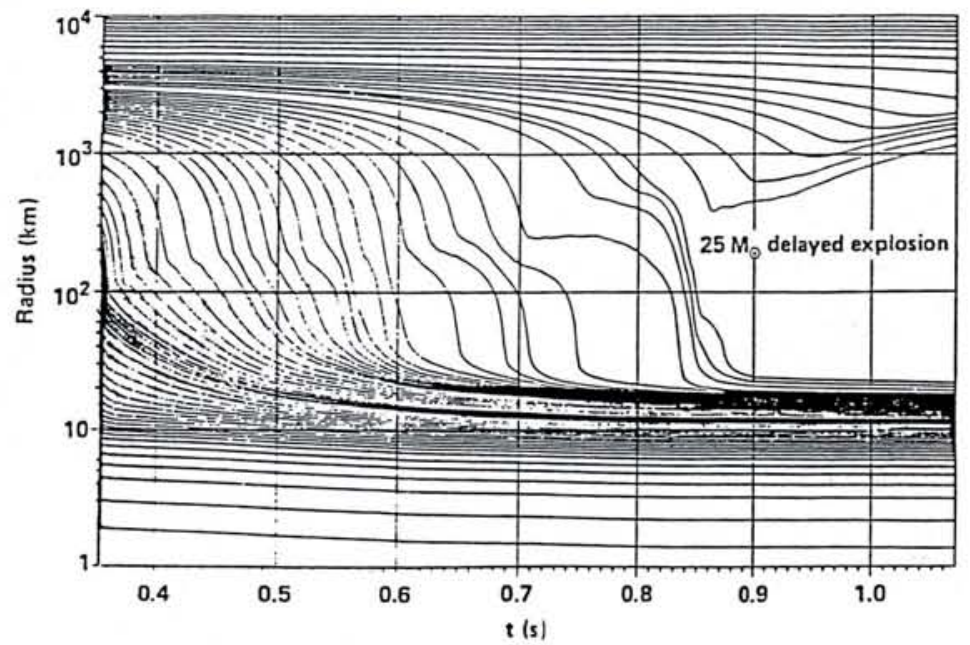

Fig. 1 - The radius of different depths as a function of time in a $25 M_{\odot}$ delayed explosion according to J.R. Wilson et al. [see ref. 2]. tons become so energetic that they are able to photodisintegrate the iron nuclei, which of course removes huge amounts of energy from the stellar centre. The central core being no longer pressure, supported undergoes collapse, which occurs at the free-fall time: within a few tens of milliseconds, the core falls from about $5000 \mathrm{~km}$ to about $20 \mathrm{~km}$, close to the final size of the resulting neutron star. The velocities reach up to 70000 $\mathrm{km} / \mathrm{s}$. The outer layers, hanging suspended, will learn what happened to the centre much later, when they will be met by the shock wave of the SN explosion, which reaches the stellar surface after three hours.

\section{Turning Collapse into Explosion}

As early as 1934, Baade and Zwicky realised that the potential energy liberated by the collapse to a neutron star is so large ( $\cong 10^{46} \mathrm{~J}$ ) that only $1 \%$ of this energy would be sufficient to remove the envelope and produce the $\mathrm{SN}$ explosion. Over the last 20 years there have been many debates over the mechanism that turns the implosion into an explosion. From recent works by Arnett, Bethe, Burrows, Lattimer, Wilson and Woosley (see ref. 1) the following picture has emerged.

When the density of the collapsing core is about three times the nuclear density, i.e. when $\rho \cong 8 \times 10^{14} \mathrm{~kg} / \mathrm{dm}^{3}$, matter becomes very stiff and a bounce occurs on the wall of the newly formed neutron star at about $0.8 M_{\odot}$ within the core. The shock energy is typically $7 \times$ $10^{44} \mathrm{~J}$, enough to make a supernova explosion. When it works, this bounce mechanism is called "prompt explosion"; it occurs nearly on the same dynamical time scale as the implosion. However, the shock wave has difficulty to make its way through the core. It loses much energy by $v$-emission and by $\mathrm{Fe}$ - photodisintegration $\left(1.5 \times 10^{45} \mathrm{~J} / \mathrm{M}_{\odot}\right)$. The shock succeeds only if the core mass is less than about $1.25 M_{\odot}$, which corresponds to a star with an initial mass of about $10 \mathrm{M}_{\odot}$.

Thus, the question arises as to how more massive stars explode. The answer was found in 1982 by J. Wilson with computer models. Nearly all the $2-3 \times$ $10^{46} \mathrm{~J}$ from the potential energy escape in the form of neutrinos. At densities below $10^{11} \mathrm{~kg} / \mathrm{dm}^{3}$, i.e. at about $20 \mathrm{~km}$ from the centre, neutrinos come out nearly freely from what is called the neutrinosphere. Within a few seconds, as much energy as 100 times the solar luminosity during 10 billion years goes out in the form of neutrinos. About one percent of this huge flux $(E=10-20$ $\mathrm{MeV}$ ) is captured by protons, neutrons and nuclei at the level of the stagnating shock at 200 to $300 \mathrm{~km}$ from the centre (cf Fig. 1). The transfer of momentum is sufficient to reverse the implosion and produce the stellar explosion. Thus, very remarkably, the neutrinos, which are the most elusive particles, produce the most violent event in Nature.

We may also emphasize that from the huge initial neutrino luminosity, only one percent is converted into kinetic energy, of which about one percent again will be converted into luminous energy of the order of $10^{42} \mathrm{~J}$. Thus, the supernova appearing in the sky is just an energetically marginal process in the birth of a neutron star. Indeed, only if the collapsing core has a mass smaller than a critical value, called the Openheimer-Volkoff mass limit, will it form a neutron star. More massive cores will further collapse to a black hole. We shall see below that $v$-measurements give interesting indications about what really did occur in SN 1987 A.

\section{Neutrino Detection}

Supernovae are the source of a thin but extremely intense $v$-shell moving through the Universe. The $v$ flux is so high that on Earth at a distance of 50000 parsec, the flux from SN $1987 \mathrm{~A}$ attained some $5 \times 10^{10} / \mathrm{cm}^{2}$ within a few seconds. Some of these neutrinos were observed in large underground experiments built to search for proton decay. The Kamiokande II detector in Japan with $2100 \mathrm{t}$ of water recorded eleven events on 23 February at 07.35.41 GMT (cf Fig. 2; ref. 5). The American IMB (Irvine - Michigan Brookhaven) experiment with $5000 \mathrm{t}$ of water observed eight events at the same time. Incidentally, we may notice that these two detectors received their neutrinos through the Earth, since SN $1987 \mathrm{~A}$ is 


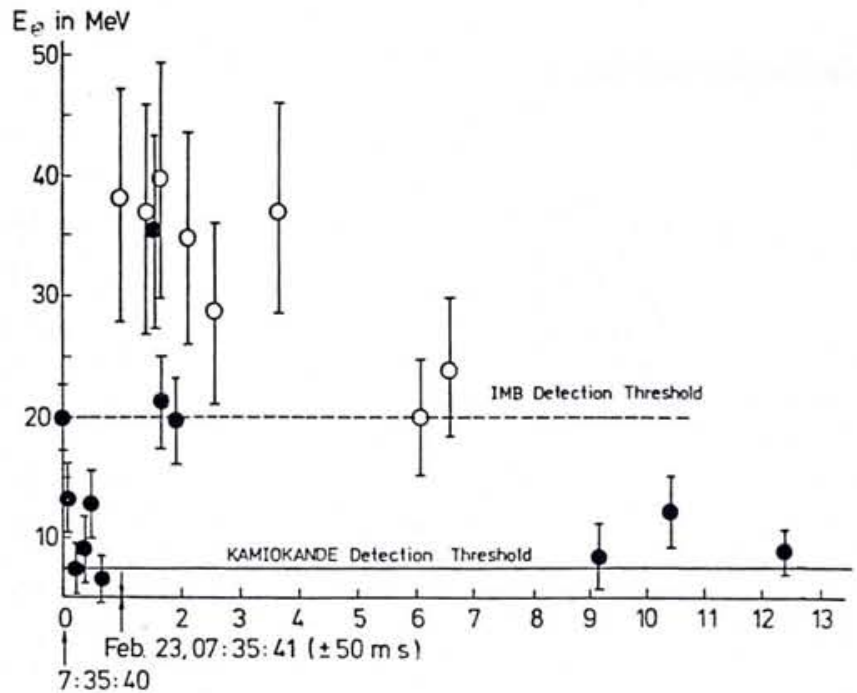

Fig. 2-The neutrino signal from SN 1987A. Filled circles: Kamiokande II; open circles: IMB [see ref. 5].

in the southern hemisphere. The dominant reaction in the water detector is $\overline{v_{e}}+p \rightarrow n+e^{+}$; Cerenkov light from pair annihilation is then observed.

Taking into account the sensitivity of the detectors, the number of $\overline{v_{e}}$ received, their energies, the fraction $1 / 6$ of $\overline{v_{e}}$ in the overall $v$ flux and the distance of the Cloud, one may estimate [cf 6] that the total $v$ energy emitted is about $2 \times$ $10^{46} \mathrm{~J}$. This corresponds remarkably to the theoretical predictions for the formation of a neutron star of $1.4 \mathrm{M}_{\odot}$. It is unlikely that a black hole has formed, the integrated $v$ signal would have been larger and exhibited an abrupt cut-off, when the general relativistic instability set in. Commenting on the beautiful results from the $v$ detection, the Texan astrophysicist D. Arnett said that up to now we had seen only the smoke from the gun; this is the first time we see the trigger pulled!

Another observation of neutrinos was made by a detector consisting of $90 \mathrm{t}$ of liquid scintillator located in the Mont Blanc tunnel: five events were recorded on 23 February at $2 \mathrm{~h} 52.34$, i.e. 4.7 hours before the other detections, whereas nothing significant was seen at the later time and the two other experiments recorded nothing at this earlier time. Several different interpretations of the alleged detection have been put forward.

Interestingly, the rather limited scatter of the arrival times of neutrinos having different energies puts an upper limit on the rest mass of the neutrinos. A reliable estimate comparing theoretical emission pattern and arrival times gives a rest mass for $\bar{v}_{\mathrm{e}}$ smaller than $12 \mathrm{eV}$ at the $90 \%$ confidence level. notice the fast decrease of the initial UV flux, already mentioned. During the first weeks, the luminous display of the $\mathrm{SN}$ was due to the shock wave, which deposits its energy into the envelope and causes it to expand; a part of this energy gradually leaks out. Were there only this energy source, the $\mathrm{SN}$ would fade out after a month or two. In Fig. 3, the light curves show a bump culminating after about 80 days when undoubtedly another energy source came into play. Although some claims have been made suggesting that the pulsar had become visible, it soon became clear that the bump and the subsequent tail of the light curve was powered by the radioactivity of ejected elements, as is well known to be the case for the tails of supernovae light curves generally. The powering radioactive source is the decay sequence involving electron capture and positron emission:

\section{Photometric and Spectroscopic Obser- vations \\ After the discovery of SN 1987 A,

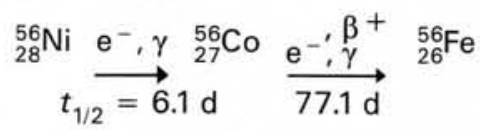
astronomers eagerly inspected the plates they had of the field. I. Shelton, the discoverer, had a plate of the same field taken the night before showing no trace of the supernova. R.H. McNaught in Australia has plates taken on $23 \mathrm{Fe}$ bruary at $10 \mathrm{~h} 38$, (i.e. three hours after the Kamiokande II and IMB detections) where the $\mathrm{SN}$ was already rising with magnitude of about 6.0. This three-hour interval corresponds well to the travel time of the shock wave between the centre and surface of the blue supergiant. For the Mont Blanc experiment, the interval would be eight hours, a value which is difficult to account for.

Fig. 3 shows the $S N$ light curves in $U$, $B, V$ colours (near UV, blue, visible). We
First, the powering comes from $\mathrm{Ni}$ and then from Co. The $\gamma$ radiation is thermalized by Compton interactions and by photoionisation at lower energies, it comes out as UV, visible and IR radiation. As the envelope expands, it becomes optically thin and thus the light curve shows after 120 days an exponential tail which just corresponds to the instantaneous radioactive power. With integrated radiation, i.e. bolometric radiation, the slope of the tail corresponds well to the half-life of $77.1 \mathrm{~d}$ of the Co radioactivity. The amount of initial $\mathrm{Ni}$ necessary to produce the observed tail intensity is estimated to be $0.07 M_{\odot}[3$, 4]; in its tail, SN 1987 A finally behaves like a normal SNII.

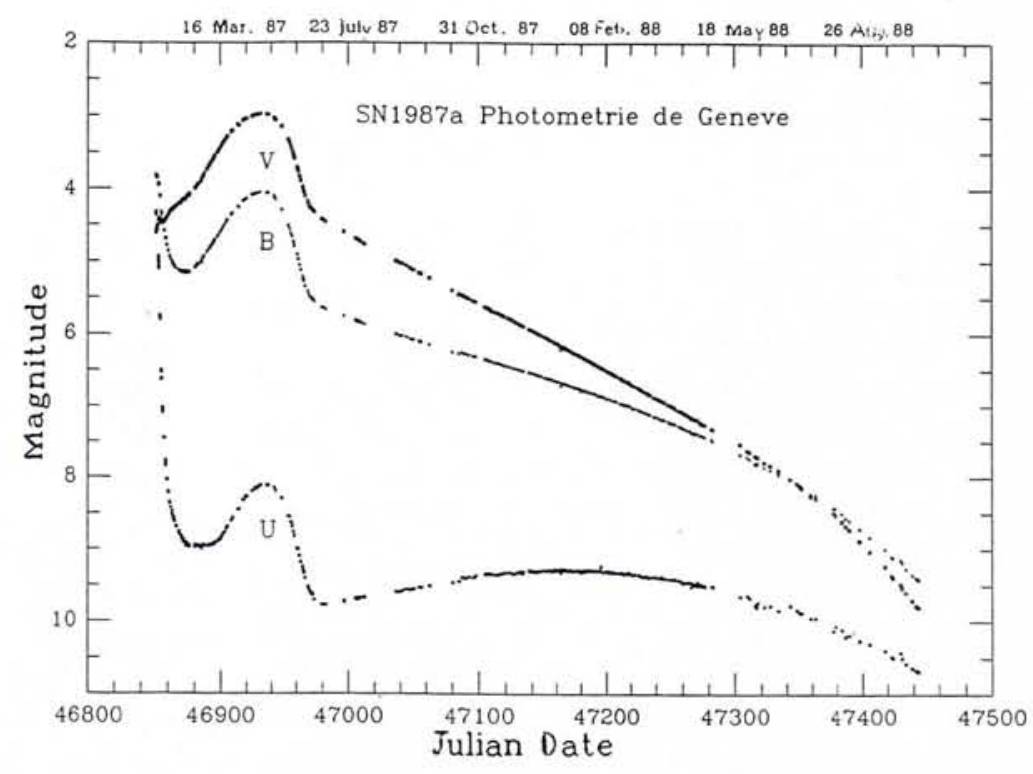

Fig. 3 - The light curve of SN 1987 A in U, B and V colours [courtesy F. Rufener]. 
As the ejected matter goes through the photosphere, the various layers of the onion skin models are revealed. As the photosphere was still in the hydrogen rich envelope, an overabundance of barium by a factor 5-10 was observed. $\mathrm{Ba}$ is a so-called s-process element, made by a succession of slow neutron captures starting from iron. This s-process has been shown to occur in the helium burning region, so the $\mathrm{Ba}$-observation in $\mathrm{H}$-rich layers seems to require some unpredicted mixing of the $\mathrm{H}$ - and $\mathrm{He}$-rich layers. Another observation suggests the same kind of effect. Very narrow absorption lines of $\mathrm{N}$-rich material were found by IUE observers. Such lines are likely to originate from matter ejected by the SN progenitor, when it was a red supergiant. The interest is that nitrogen is a product of the $\mathrm{C}, \mathrm{N}, \mathrm{O}$ burning of hydrogen and that such an $\mathrm{N}$-excess (factor 30) indicates that a large amount of these products have been brought to the stellar surface during the precursor evolution either by unpredicted efficient mixing processes or as a result of very heavy mass loss.

From IR observations with the Kuiper Airborne Observatory, material from the inner core started to be visible in November 1987. Lines of the elements $\mathrm{Ni}, \mathrm{Ar}$, Co appeared with strengths indicating abundances far higher than normal, which implies that freshly synthesized elements were seen. This stellar striptease is receiving the greatest attention from astrophysicists, who have thus an invaluable opportunity to test the predictions of the models for stellar nucleosynthesis.

\section{$X$ and $\gamma$-rays from SN 1987 A}

Radioactive cobalt emits two $\gamma$ lines, at 847 and $1238 \mathrm{keV}$, which are powering the tail of the optical light curve. The first theoretical models predicted that these $\gamma$-rays should appear as degraded photons in the range of $10-200 \mathrm{keV}$ about a year after the explosion. Indeed, the Ginga satellite $(10-30 \mathrm{keV})$ and the Kvant-Roentgen experiment $\left(15-10^{3}\right.$ $\mathrm{keV}$ ) on the Mir mission detected X-rays already in the middle of August 1987. The shape of the X-spectrum corresponds well to the theoretical predictions [7]. The early arrival of X-rays was interpreted $[3,4]$ as the result of the mixing of radioactive $\mathrm{Ni}$ and $\mathrm{Co}$ during the explosion. The explosion might have been non radial or might have had "fingers", alternatively a Rayleigh-Taylor instability due to the radiation of $\mathrm{Ni}$ and $\mathrm{Co}$ might have occurred and produced the necessary mixing during the explosion - to quote some of the proposed explanations. The $\gamma$-detectors on the SMM satellite (Solar Maximum Mission) and on some balloon flights observed the 847 and $1238 \mathrm{keV}$ lines already from November 1987. This appearance is also early and interpreted as indicative of some substantial mixing in the explosion.

Interestingly enough, the soft $\mathrm{X}$-ray component below $10 \mathrm{keV}$ is time-variable. The origin of this variability is not exactly known and has been attributed either to clumps in the ejected matter or to contributions from the synchrotron radiation of the buried pulsar, supposed to be seen through holes and irregularities in the ejecta.

What comes out directly in the form of $X$-and $\gamma$-rays will no longer contribute to the UV, visible and IR radiation. This is certainly the main reason for the turning down of the bolometric light curve after May 88; this trend is also well visible on the V-curve in Fig. 3. On the other hand, the luminous contribution from a central pulsar could introduce upward deviations from the linear tail of the curve. As the emitted power of a pulsar goes as $\Omega^{-4}$, where $\Omega$ is the angular velocity of the rotating neutron star, it is clear that we cannot anticipate what the pulsar luminosity will be, since we do not know its rotation rate. At the time of writing, there is still no indication about the supposed central pulsar. Thus, after the numerous beautiful observations already offered by SN 1987 A, the astronomical community realizes that many promising results of this fascinating supernova are still ahead from us.

\section{REFERENCES}

[1] Woosley S.E. and Weaver T.A., Ann. Rev. Astron. Astrophys. 24 (1986) 205.

[2] Woosley S.E., Nucleosynthesis and Stellar Evolution, 16th Advanced Course of the Swiss Society of Astron. and Astrophys., Ed. B. Hauck et al., Geneva Observatory, p. 1.

[3] Nomoto K., Shigeyama T. and Hashimoto M., Lecture Notes in Physics 305 (1988) 319.

[4] Woosley S.E. ibid. 361.

[5] Koshiba M., in 'SN 1987 A', ESO Workshop, Ed. I.J. Danziger, ESO Proceedings (1987) 219.

[6] Burrows A. ibid. 315.

[7] Sunyaev R. et al., Nature 330 (1987) 227.

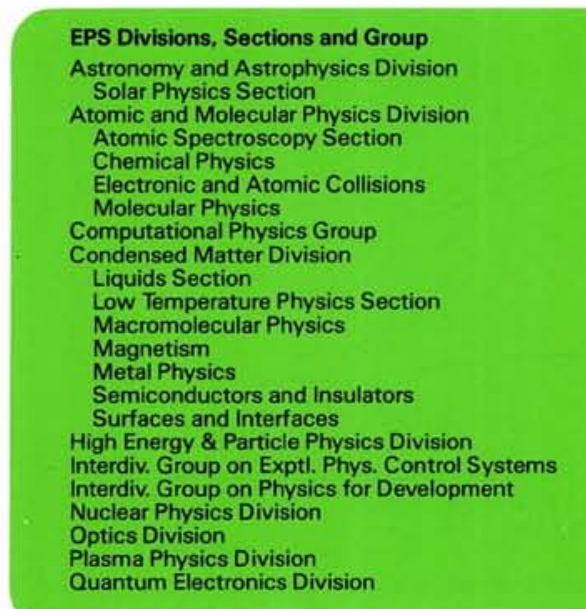

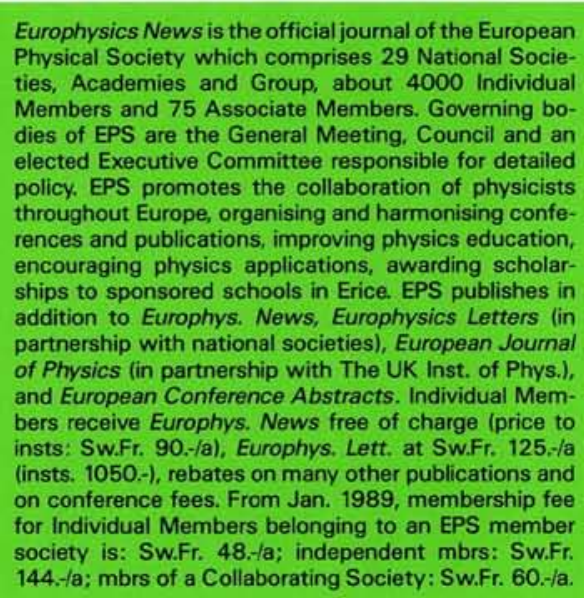

\section{Kurti Hands Over to Buckel}

During the long discussions that led to the creation of Europhysics Letters no topic was more thoroughly argued than that of the first editor. He clearly had to be an eminent physicist, highly articulate, a person of great experience and with a wide European background, an accomplished diplomat, a shrewd judge, unassailable good humour and tough - physically as well as mentally. Mixing so many ingredients into one individual it was eventually realised, required also the skills of a maître cuisinier - and so the choice fell naturally on Nicolas Kurti of Oxford. Three years and eight volumes later, each one a banquet in itself, the time has come for him to retire and hand over to a new chef. The European Physical Society is happy to announce that former President Werner Buckel has agreed to don the hat of Editor in Chief from 1 April 1989.

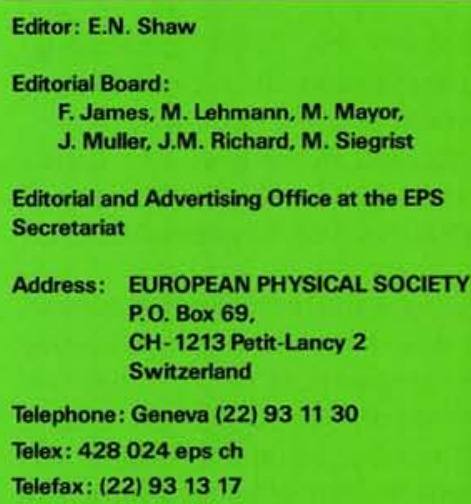

